\title{
New Variables of Separation for the Steklov-Lyapunov System
}

Andrey V. TSIGANOV

St. Petersburg State University, St. Petersburg, Russia

E-mail: andrey.tsiganov@gmail.com

Received October 31, 2011, in final form March 12, 2012; Published online March 20, 2012

http://dx.doi.org/10.3842/SIGMA.2012.012

\begin{abstract}
A rigid body in an ideal fluid is an important example of Hamiltonian systems on a dual to the semidirect product Lie algebra $e(3)=s o(3) \ltimes \mathbb{R}^{3}$. We present the biHamiltonian structure and the corresponding variables of separation on this phase space for the Steklov-Lyapunov system and it's gyrostatic deformation.
\end{abstract}

Key words: bi-Hamiltonian geometry; variables of separation

2010 Mathematics Subject Classification: 70H20; 70H06; 37K10

\section{Introduction}

Rigid body dynamics in an ideal incompressible fluid is rich with problems interesting from mathematical point of view, in particular, the research of integrable problems. Certainly, the most famous are the three integrable cases under the names of Kirchhoff, Clebsch, Steklov and Lyapunov. The latter two cases are more interesting because there are no a obvious symmetry groups associated with the additional integrals of motion.

All these classical cases were discovered and carefully studied in the 18th and 19th centuries [5]. For instance, the Kirchhoff equations for the Clebsch and Steklov-Lyapunov cases were first solved explicitly by Kötter after some mysterious separation of variables [11, 12]. At the moment no separation which is alternative to his original separation of variables is known for these systems, even though there is a lot of literature dedicated to the problem, including the theta-functions solutions, associated with the Lax matrices, and the detailed geometric descriptions of the invariant surfaces on which the motions evolve, see books $[1,2]$ and references within.

In this paper we apply the bi-Hamiltonian geometry to the direct calculation of variables of new separation for the Steklov-Lyapunov system and it's Rubanovsky generalization. We have to notice right away, that our main purpose is the development of the bi-Hamiltonian geometry instead of integration of particular equations of motion using separation of variables method.

An integrable system is separable, if there are $n$ separation relations

$$
\Phi_{i}\left(u_{i}, p_{u_{i}}, H_{1}, \ldots, H_{n}\right)=0, \quad i=1, \ldots, n, \quad \text { with } \quad \operatorname{det}\left[\frac{\partial \Phi_{i}}{\partial H_{j}}\right] \neq 0,
$$

connecting single pairs $\left(u_{i}, p_{u_{i}}\right)$ of canonical variables of separation with the $n$ functionally independent Hamiltonians $H_{1}, \ldots, H_{n}$. Solving these relations in terms of $p_{u_{i}}$ one gets the Jacobi equations and the corresponding additively separable complete integral of the Hamilton-Jacobi equation

$$
W=\sum_{i=1}^{n} \int^{u_{i}} p_{u_{i}}\left(u_{i}^{\prime}, \alpha_{1}, \ldots, \alpha_{n}\right) d u_{i}^{\prime}, \quad \alpha_{j}=H_{j} .
$$


Of course, any variables of separation are determined up to the trivial transformation

$$
u_{i} \rightarrow \tilde{u}_{i}=f_{i}\left(u_{i}, p_{u_{i}}\right),
$$

which preserve all the properties of algebraic curves defined by separated relations (1.1). However, if we have another separated relations for the same integrals of motion

$$
\Psi_{i}\left(v_{i}, p_{v_{i}}, H_{1}, \ldots, H_{n}\right)=0, \quad i=1, \ldots, n, \quad \text { with } \quad \operatorname{det}\left[\frac{\partial \Psi_{i}}{\partial H_{j}}\right] \neq 0,
$$

which can not be reduced to initial ones (1.1) by trivial change of variables (1.2), we usually say about different variables of separation. Of course, any two families of canonical variables on a given phase space are related by a generic canonical transformations

$$
v_{i}=g_{i}\left(u_{1}, \ldots, u_{n}, p_{1}, \ldots, p_{n}\right), \quad p_{v_{i}}=h_{i}\left(u_{1}, \ldots, u_{n}, p_{1}, \ldots, p_{n}\right) .
$$

In contrast with (1.2) the notion of generic transformations (1.3) allows us to study relations between distinct algebraic curves, for instance covering of the algebraic curves (see works of Poincaré, Humbert, Frey, Kani, Kuhn, Shaska) or curves with isogenous Abel varietes (see works of Richelot, Brock, Hayashida, Nishi, Ibukiyama, Katsura, van Wamelen). Such relations give us a lot of examples of the reductions of Abelian integrals (see works of Hermite, Goursat, Burkhardt, Brioschi, Bolza) and, therefore, they may be a source of new ideas in number theory, algebraic geometry and modern cryptography (see works of Tate, Faltings, Zarhin, Lange, McMullen, Merel).

Nevertheless, motivation for the search of such different variables of separation do not pure mathematical, because different variables of separation may be useful in different perturbation theories [18, 22, 24], as well as in distinct procedures of quantization and various methods of qualitative analysis, etc.

The milestones of the variables separation technique include the works of Stäckel, LeviCivita, Eisenhart, Benenti and others. The majority of results was obtained for a very special class of integrable systems, important from the physical point of view, namely for the natural Hamiltonian systems with quadratic in momenta integrals of motion on cotangent bundles to Riemannian manifolds. The Kowalevski and Chaplygin results on separation of variables for the systems with higher-order integrals of motion have been missed out of this scheme until recently $[18,22,24]$.

In the Steklov-Lyapunov case we have quadratic integrals of motions, but the phase space is the Poisson manifold instead of the cotangent bundle to Riemannian manifold. So, in this case we can use neither the Levi-Civita criteria, nor the Eisenhart-Benenti theory. Below we show how variables of separation for the given integrable system may be calculated without any additional information (Killing tensors, Lax matrices, $r$-matrices, links with soliton equations etc.).

\section{Steklov-Lyapunov system}

Following Kirchhoff, we consider the potential motion of a finite rigid body submerged in an infinitely large volume of irrotational, incompressible, inviscid fluid that is at rest at infinity, so that the induced motion of particles of the fluid is completely determined by the motion of the body [9]. In this case, the motion of rigid body is described by the classical Kirchhoff equations

$$
\dot{M}=M \times \Omega+p \times U, \quad \dot{p}=p \times \Omega,
$$

here $x \times y$ stands for the vector product of three-dimensional vectors. Vectors $M$ and $p$ are the impulsive momentum and the impulsive force while $\Omega$ and $U$ are the angular and linear 
velocities of the body. All these vectors in $\mathbb{R}^{3}$ are expressed in the body frame attached to the body originating at the center of buoyancy [9].

A rigid body in the ideal fluid is an important example of Hamiltonian systems on a dual to Lie algebra $e(3)=s o(3) \ltimes \mathbb{R}^{3}$. The dual space $e^{*}(3)$ is the Poisson manifold endowed with the canonical Lie-Poisson brackets

$$
\left\{M_{i}, M_{j}\right\}=\varepsilon_{i j k} M_{k}, \quad\left\{M_{i}, p_{j}\right\}=\varepsilon_{i j k} p_{k}, \quad\left\{p_{i}, p_{j}\right\}=0
$$

where $\varepsilon_{i j k}$ is a totally skew-symmetric tensor. There are two Casimir elements

$$
C_{1}=\langle p, p\rangle=|p|^{2} \equiv \sum_{i=1}^{3} p_{i}^{2}, \quad C_{2}=\langle p, M\rangle \equiv \sum_{i=1}^{3} p_{i} M_{i}
$$

where $\langle x, y\rangle$ means scalar product of two three-dimensional vectors $x, y \in \mathbb{R}^{3}$.

As usual [9], element $M \in s o(3)$ is identified with three-dimensional vector $M \in \mathbb{R}^{3}$ using well known isomorphism of the Lie algebras $\left(\mathbb{R}^{3}, \times\right)$ and $s o(3),[\cdot, \cdot]$

$$
z=\left(z_{1}, z_{2}, z_{3}\right) \rightarrow z_{\mu}=\left(\begin{array}{ccc}
0 & z_{3} & -z_{2} \\
-z_{3} & 0 & z_{1} \\
z_{2} & -z_{1} & 0
\end{array}\right)
$$

where $\times$ is a cross product, $[\cdot, \cdot]$ is a matrix commutator and index $\mu$ means a $3 \times 3$ antisymmetric matrix associated with the vector $z$. Using this agreement we can rewrite canonical Poisson bivector on $e^{*}(3)$ in the following compact form

$$
P=\left(\begin{array}{cccccc}
0 & 0 & 0 & 0 & p_{3} & -p_{2} \\
0 & 0 & 0 & -p_{3} & 0 & p_{1} \\
0 & 0 & 0 & p_{2} & -p_{1} & 0 \\
0 & p_{3} & -p_{2} & 0 & M_{3} & -M_{2} \\
-p_{3} & 0 & p_{1} & -M_{3} & 0 & M_{1} \\
p_{2} & -p_{1} & 0 & M_{1} & -M_{1} & 0
\end{array}\right)=\left(\begin{array}{cc}
0 & p_{\mu} \\
p_{\mu} & M_{\mu}
\end{array}\right) .
$$

The Hamilton function $H=H(p, M)$ and Lie-Poisson brackets (2.2) allow us to define the Hamiltonian equations of motion

$$
\dot{M}=M \times \frac{\partial H}{\partial M}+p \times \frac{\partial H}{\partial p}, \quad \dot{p}=p \times \frac{\partial H}{\partial M} .
$$

These generic Euler's equations on $e^{*}(3)$ coincide with the Kirchhoff equations $(2.1)$, if $H(p, M)$ is a second-order polynomial in variables $M$ and $p$.

Remark 1. The Lie-Poisson dynamics on $e^{*}(3)$ can be interpreted as resulting from reduction by the symmetry group $E(3)$ of the full dynamics on the twelve-dimensional phase space $T^{*} E(3)$. Here, symmetry means that the Hamiltonian that describes the dynamics in $T^{*} E(3)$ is an invariant to actions of $E(3)$, i.e., one can translate the inertial frame or rotate it in any direction without affecting the equations of motion [15].

The Steklov-Lyapunov case of the rigid body motion is characterized by the following secondorder homogeneous polynomial integrals of motion

$$
\begin{aligned}
& H_{1}=\langle M, M\rangle-2\langle\mathbf{A} p, M\rangle-\left\langle\left(\mathbf{A}^{2}+2 \mathbf{A}^{\vee}\right) p, p\right\rangle, \\
& H_{2}=\langle\mathbf{A} M, M\rangle+2\left\langle\mathbf{A}^{\vee} p, M\right\rangle-\left(\left\langle\mathbf{A}^{3} p, p\right\rangle-\operatorname{tr} \mathbf{A}^{2}\langle\mathbf{A} p, p\rangle\right),
\end{aligned}
$$


where wedge denotes an adjoint matrix, i.e. a cofactor matrix $\mathbf{A}^{\vee}=(\operatorname{det} \mathbf{A}) \mathbf{A}^{-1}$. For integrability $\mathbf{A}$ has to be symmetric matrix, which may be reduced to the diagonal form

$$
\mathbf{A}=\operatorname{diag}\left(a_{1}, a_{2}, a_{3}\right), \quad a_{i} \in \mathbb{R}
$$

using linear canonical transformations of $e^{*}(3)$. From physical point of view it means that the body axes can always be chosen so that $\mathbf{A}$ is diagonal.

Remark 2. In [17] Steklov found integrable Hamiltonian $H_{2}$, whereas Lyapunov proved integrability of the Kirchhoff equations with Hamiltonian $H_{1}$ in [14]. The generic family of the Steklov-Lyapunov integrable systems was studied by Kolosov in [10].

\subsection{Separation of variables by Kötter}

The explicit integration of the classical Steklov-Lyapunov systems via separation of variables had been first made by F. Kötter in 1900 [11]. Here we want to add some new details in the known coincidence of the Kötter variables of separation $v_{1,2}$ with the elliptic coordinates on the sphere.

According to $[23,25]$ there is a Poisson map, which identifies the Steklov-Lyapunov system with a system that describes motion on the surface of a unit two-dimensional sphere $\mathbb{S}^{2}$ in a fourth-degree polynomial potential field. This dynamical system is separable in standard elliptic coordinates on the sphere, and the inverse Poisson map allows us to get complete solution of the Steklov-Lyapunov system.

Proposition 1. If $\mathbf{B}=\operatorname{tr} \mathbf{A}-\mathbf{A}$ and $\mathbf{C}=\sqrt{\mathbf{B}^{2}-4 \mathbf{A}^{\vee}}$ are diagonal matrices with entries

$$
\mathbf{B}=\left(\begin{array}{ccc}
a_{2}+a_{3} & 0 & 0 \\
0 & a_{1}+a_{3} & 0 \\
0 & 0 & a_{1}+a_{2}
\end{array}\right), \quad \mathbf{C}=\left(\begin{array}{ccc}
a_{2}-a_{3} & 0 & 0 \\
0 & a_{3}-a_{1} & 0 \\
0 & 0 & a_{1}-a_{2}
\end{array}\right)
$$

then the Poisson map $f:(p, M) \rightarrow(x, J)$, defined by

$$
x=\frac{(M-\mathbf{B} p) \times p}{|(M-\mathbf{B} p) \times p|}, \quad J=M+\mathbf{C}[x, x \times p]_{+},
$$

where

$$
[y, z]_{+i}=\sum_{j, k=1}^{n=3}\left|\varepsilon_{i j k}\right| y_{j} z_{k}
$$

relates manifold $e^{*}(3)$ with coordinates $(p, M)$ and cotangent bundle $T^{*} \mathbb{S}^{2}$ to the unit twodimensional sphere $\mathbb{S}^{2}$ with coordinates $(x, J)$.

The proof consists in the verification of the Lie-Poisson brackets between variables $x$ and $J$

$$
\left\{J_{i}, J_{j}\right\}=\varepsilon_{i j k} J_{k}, \quad\left\{J_{i}, x_{j}\right\}=\varepsilon_{i j k} x_{k}, \quad\left\{x_{i}, x_{j}\right\}=0,
$$

and calculation of the corresponding Casimir functions

$$
\langle x, x\rangle=|x|^{2}=1, \quad \text { and } \quad\langle x, J\rangle=0 .
$$

So, this coajoint orbit of $e^{*}(3)$ with coordinates $x$ and $J$ is simplectomorphic to cotangent bundle $T^{*} \mathbb{S}^{2}$ to the unit two-dimensional sphere $\mathbb{S}^{2}$, see for instance [15]. 
Inverse Poisson map $f^{-1}:(x, J) \rightarrow(p, M)$ looks like

$$
p=\alpha J+\beta(x \times J), \quad M=J-\mathbf{C}[x, x \times p]_{+},
$$

where functions $\alpha, \beta$ on $x, J$ are solutions of the following equations

$$
\begin{aligned}
C_{1}=\langle p, p\rangle= & \alpha^{2}|J|^{2}+\beta^{2}|x \times J|^{2}, \\
C_{2}=\langle p, M\rangle= & \alpha^{2}\left(|J|^{2}\langle x, \mathbf{A} x\rangle-\langle(x \times J), \mathbf{A}(x \times J)\rangle\right)+2 \alpha \beta\langle J, \mathbf{A}(x \times J)\rangle \\
& +\beta^{2}(\langle(x \times J), \mathbf{B}(x \times J)\rangle-2\langle J, \mathbf{A} J\rangle)+\alpha|J|^{2} .
\end{aligned}
$$

Proposition 2. The Poisson map (2.9) relates the Steklov integral of motion $H_{1}(p, M)$ with the natural Hamilton function on $T^{*} \mathbb{S}^{2}$

$$
\begin{aligned}
H_{1}(x, J)= & \langle J, J\rangle+4\langle x, \mathbf{B} x\rangle\left(\left(\operatorname{tr} \mathbf{A} C_{1}-C_{2}\right)-C_{1}\langle x, \mathbf{B} x\rangle\right)+4 C_{1}\left\langle x, \mathbf{A}^{\vee} x\right\rangle \\
& +2 \operatorname{tr} \mathbf{A} C_{2}-\operatorname{tr}\left(\mathbf{A}^{2}+4 \mathbf{A}^{\vee}\right) C_{1} .
\end{aligned}
$$

The Lyapunov integral $\mathrm{H}_{2}(p, M)(2.7)$ is equal to

$$
\begin{aligned}
H_{2}(x, J)= & \langle J, \mathbf{A} J\rangle-4\left\langle x, \mathbf{A}^{\vee} x\right\rangle\left(C_{1}\langle x, \mathbf{B} x\rangle-\left(\operatorname{tr} \mathbf{A} C_{1}-C_{2}\right)\right) \\
& +2 \operatorname{tr} \mathbf{A} C_{2}-2 \operatorname{det} \mathbf{A} C_{1} .
\end{aligned}
$$

Here $C_{1,2}$ are values of the Casimir functions (2.3).

The proof of this proposition and all the details may be found in [23, 25].

Remark 3. It is well-known that the generic level sets of the Casimir functions (coadjoint orbits) on $e^{*}(3)$ are only diffeomorphic to the cotangent bundle $T^{*} \mathbb{S}^{2}$. It allows us to directly connect the Kirchhoff equations with the equations of motion by geodesic on $\mathbb{S}^{2}$, however, at $C_{2} \neq 0$ we have to destroy the standard symplectic structure on $T^{*} \mathbb{S}^{2}$ by adding some "monopole" terms [15].

In the Steklov-Lyapunov case we use the Poisson map (2.9), which preserves the standard symplectic structure on $T^{*} \mathbb{S}^{2}$. As a punishment for this preservation of the standard symplectic structure we have to consider potential motion on $\mathbb{S}^{2}$ instead of the geodesic motion.

The Hamiltonians $H_{1,2}(x, J)(2.11),(2.12)$ on $T^{*} \mathbb{S}^{2}$ are separable in the elliptic (spheroconical) coordinates $v_{1,2}$, which are zeroes of the function

$$
e(\lambda)=\frac{\left(\lambda-v_{1}\right)\left(\lambda-v_{2}\right)}{\operatorname{det}(\lambda-\mathbf{A})}=\left\langle x,(\lambda-\mathbf{A})^{-1} x\right\rangle \equiv \sum_{i=1}^{3} \frac{x_{i}^{2}}{\lambda-a_{i}} .
$$

This variables of separation satisfy the following separated relations

$$
\Phi\left(v_{i}, p_{v_{i}}\right)=\operatorname{det}\left(v_{i}-\mathbf{A}\right) p_{v_{i}}^{2}+\left\langle\ell\left(v_{i}\right), \ell\left(v_{i}\right)\right\rangle=0, \quad i=1,2,
$$

where the three-dimensional vector $\ell(\lambda)$ is the so-called Kötter vector with entries

$$
\ell_{i}(\lambda)=\frac{\sqrt{\lambda-a_{i}}}{2}\left(M_{i}+\left(2 \lambda+a_{i}-\operatorname{tr} \mathbf{A}\right) p_{i}\right)
$$

depending on the auxiliary variable $\lambda$ (spectral parameter). The explicit solution of the corresponding Abel-Jacobi equations in theta-functions was given in [11]. In order get initial variables $p, M$ as functions on time variable we have to substitute solutions of the Abel-Jacobi equations into the variables on $T^{*} \mathbb{S}^{2}$

$$
x_{i}=\sqrt{\frac{\left(v_{1}-a_{i}\right)\left(v_{2}-a_{i}\right)}{\left(a_{j}-a_{i}\right)\left(a_{k}-a_{i}\right)}}, \quad J_{i}=\frac{2 \varepsilon_{i j k} x_{j} x_{k}\left(a_{j}-a_{k}\right)}{v_{1}-v_{2}}\left(\left(a_{i}-v_{1}\right) p_{v_{1}}-\left(a_{i}-v_{2}\right) p_{v_{2}}\right),
$$

where $(i, j, k)$ is permutation of $(1,2,3)$, and then into the variables $p$ and $M$ (2.10). Another modern verification of the Kötters calculations may be found in $[4,8]$. 


\section{Calculation of the variables of separation in bi-Hamiltonian geometry}

We can only guess how Kötter invented the variables of separation $v_{1,2}$, which coincide with the elliptic coordinates on the auxiliary two-dimensional sphere, because he gave no explanations of calculations in very brief communication [11]. It is clear that behind the striking formulas there must be a certain geometric idea, but the domain of applicability of this idea is usually restricted by a partial model under consideration. For instance, we can not apply the Kötter separation to the Kowalevski top and Kowalevski separation to the Steklov-Lyapunov system etc.

Our aim is to discuss some algorithm of calculation of the variables of separation in the framework of the bi-Hamiltonian geometry, which is applicable to many known integrable systems $[7,18,20,21,22,24,26]$. In fact this algorithm consists of the following steps:

- calculate the second Poisson bracket compatible with canonical one starting with the given integrals of motion in the involution with respect to this canonical Poisson bracket;

- if the Poisson brackets have different symplectic leaves, calculate a projection of the second bracket on symplectic leaves of the first bracket;

- calculate coordinates of separation as eigenvalues of the corresponding recursion operator;

- calculate the canonically conjugated momenta with respect to the first Poisson bracket;

- calculate the separated relations.

The input of algorithm is a set of integrals of motion and canonical Poisson bracket, whereas output is a set of separated relations. All details about construction of a suitable projection are discussed in [7].

Because variables of separation (1.1) are defined up to canonical transformations $u_{i} \rightarrow$ $f\left(u_{i}, p_{u_{i}}\right)$ on the first step of this algorithm we have to narrow the search space using some artificial tricks. It is a main technical problem of this method. The second technical problem is the calculation of the momenta conjugated to obtained coordinates, see [7, 18, 22, 24].

\subsection{Polynomial and rational Poisson brackets on $e^{*}(3)$}

Bi-Hamiltonian structures can be seen as a dual formulation of integrability and separability, in the sense that they substitute a hierarchy of compatible Poisson structures to the hierarchy of functions in involution, which may be treated either as integrals of motion or as variables of separation. So, our first step is calculation of the second Poisson bivector $P^{\prime}$ compatible with kinematic Poisson bivector $P$.

According to $[20,26]$ any separable system is a bi-integrable system, i.e. integrals of motion $H_{k}(1.1)$ are in bi-involution

$$
\left\{H_{i}, H_{k}\right\}=\left\{H_{i}, H_{k}\right\}^{\prime}=0 v, \quad i, k=1, \ldots, n,
$$

with respect to compatible Poisson brackets $\{\cdot, \cdot\}$ and $\{\cdot, \cdot\}^{\prime}$ associated with the Poisson bivectors $P$ and $P^{\prime}$, so that

$$
\llbracket P, P \rrbracket=0, \quad \llbracket P, P^{\prime} \rrbracket=0, \quad \llbracket P^{\prime}, P^{\prime} \rrbracket=0 .
$$

Here $\llbracket \cdot, \cdot \rrbracket$ is the Schouten bracket. The definition of the second bracket $\{\cdot, \cdot\}^{\prime}$ in term of variables of separation may be found in $[20,26]$.

For the given integrable system fixed by a kinematic bivector $P$ and a tuple of integrals of motion $H_{1}, \ldots, H_{n}$ bi-Hamiltonian construction of variables of separation consists in a direct 
solution of the equations (3.1) and (3.2) with respect to an unknown bivector $P^{\prime}$. The main problem is that the geometrically invariant equations (3.1), (3.2) have a'priory infinite number of solutions [18, 20, 21, 22, 24, 26].

In order to get a search algorithm of effectively computable solutions we have to narrow the search space by using some non-invariant additional assumptions. According to [20, 21] hereafter we assume that $P^{\prime}$ has the same foliations by symplectic leaves as $P$, i.e. that

$$
P^{\prime} d C_{1,2}=0
$$

and $P^{\prime}$ doesn't have any other Casimir elements. The geometric meaning of this restriction is discussed in [20, 21]. In fact it allows us to avoid calculations of the projection of the second bracket on the symplectic leaves of the first bracket.

In the Steklov-Lyapunov case solving equations (3.1), (3.2) and (3.3) in the space of homogeneous second-order polynomial bivectors and of rational bivectors with the second-order homogeneous numerators and linear denominators we obtain the following two propositions.

Proposition 3. If $c$ and $d$ are two numeric three-dimensional vectors, so that $\langle c, c\rangle=0$, then equations (3.2) and (3.3) on $e^{*}(3)$ have a polynomial solution

$$
P_{1}^{\prime}=\left(\begin{array}{cc}
\langle c, p\rangle p_{\mu} & \langle c, M\rangle p_{\mu}+(p \times M) \otimes c+\frac{1}{2}\left(\frac{1}{\alpha}+\alpha\langle c, d\rangle\right)(p \otimes p-\langle p, p\rangle) \\
* & \\
* & \\
& \langle c, M\rangle M_{\mu}+\langle d \times p) \otimes(d \times p) \\
& -\alpha\left((c \times p) \times(d \times M) p_{\mu}+\frac{1}{2}\left(\frac{1}{\alpha}+\alpha\langle c, d\rangle\right)(p \times M)_{\mu}\right.
\end{array}\right),
$$

$\alpha \in \mathbb{C}$, and a rational solution

$$
P_{2}^{\prime}=\frac{1}{\langle c, p\rangle} P_{1}^{\prime}+\frac{1}{\langle c, p\rangle}\left(\langle c, M\rangle+\frac{\alpha\left(1+\alpha^{2}\langle c, d\rangle\right)}{2 \alpha^{2}\langle c, d\rangle}\langle c \times p, d\rangle\right) P
$$

compatible to each other, i.e. $\llbracket P_{1}^{\prime}, P_{2}^{\prime} \rrbracket=0$.

As above $\times$ is a cross product, the antisymmetric matrix $z_{\mu}$ is defined by vector $z(2.4)$ and the matrix $(x \otimes y)_{i j}=x_{i} y_{j}$ is determined by a pair of vectors $x$ and $y$. In order to explain this notations we write out the corresponding Poisson brackets

$$
\begin{aligned}
\left\{p_{i}, p_{j}\right\}_{1}^{\prime}= & \varepsilon_{i j k}\langle c, p\rangle p_{k}, \\
\left\{p_{i}, M_{j}\right\}_{1}^{\prime}= & \varepsilon_{i j k}\langle c, M\rangle p_{k}+(p \times M)_{i} c_{j}+\frac{1}{2 \alpha}\left(1+\alpha^{2}\langle c, d\rangle\right)\left(p_{i} p_{j}-\sum_{l=1}^{3} p_{l}^{2}\right) \\
& +\alpha(c \times p)_{i}(d \times p)_{j}, \\
\left\{M_{i}, M_{j}\right\}_{1}^{\prime}= & \varepsilon_{i j k}\left(\langle c, M\rangle M_{k}+\langle d, p\rangle p_{k}\right) \\
& +\varepsilon_{i j k}\left(\frac{1}{2 \alpha}\left(1+\alpha^{2}\langle c, d\rangle\right)(p \times M)_{k}-\alpha((c \times p) \times(d \times M))_{k}\right) .
\end{aligned}
$$

The second brackets are equal to

$$
\begin{aligned}
& \left\{p_{i}, p_{j}\right\}_{2}^{\prime}=\varepsilon_{i j k} p_{k}, \\
& \left\{p_{i}, M_{j}\right\}_{2}^{\prime}=\frac{\left\{p_{i}, M_{j}\right\}_{1}^{\prime}}{\langle c, p\rangle}+\frac{\varepsilon_{i j k} p_{k}}{\langle c, p\rangle}\left(\langle c, M\rangle+\frac{\alpha\left(1+\alpha^{2}\langle c, d\rangle\right)}{2 \alpha^{2}\langle c, d\rangle}\langle c \times p, d\rangle\right), \\
& \left\{M_{i}, M_{j}\right\}_{2}^{\prime}=\frac{\left\{M_{i}, M_{j}\right\}_{1}^{\prime}}{\langle c, p\rangle}+\frac{\varepsilon_{i j k} M_{k}}{\langle c, p\rangle}\left(\langle c, M\rangle+\frac{\alpha\left(1+\alpha^{2}\langle c, d\rangle\right)}{2 \alpha^{2}\langle c, d\rangle}\langle c \times p, d\rangle\right) .
\end{aligned}
$$

We have to stress that this brackets are defined over complex field because $\langle c, c\rangle=c_{1}^{2}+c_{2}^{2}+c_{3}^{2}=0$. 
Substituting the Poisson brackets $\{\cdot, \cdot\}_{1,2}^{\prime}(3.6)$, (3.7) into (3.1) and solving the resulting equations in the space of the second-order homogeneous polynomials $H_{1,2}$ one gets the following proposition.

Proposition 4. The Steklov-Lyapunov integrals of motion $H_{1,2}(p, M)(2.7)$ satisfy the equation (3.1) at $\alpha=1$ and

$$
\begin{aligned}
& c=\frac{-1}{\sqrt{\left(a_{1}-a_{2}\right)\left(a_{2}-a_{3}\right)\left(a_{3}-a_{1}\right)}}\left(\sqrt{a_{2}-a_{3}}, \sqrt{a_{3}-a_{1}}, \sqrt{a_{1}-a_{2}}\right), \\
& d=\sqrt{\left(a_{1}-a_{2}\right)\left(a_{2}-a_{3}\right)\left(a_{3}-a_{1}\right)}\left(\frac{1}{\sqrt{a_{2}-a_{3}}}, \frac{1}{\sqrt{a_{3}-a_{1}}}, \frac{1}{\sqrt{a_{1}-a_{2}}}\right) .
\end{aligned}
$$

The proof is a straightforward calculation.

Remark 4. In fact, polynomial bivector $P_{1}^{\prime}$ has been obtained in [21] as an incidental result by investigation of the Poisson bivectors on the Lie algebra $s o^{*}(4)$ and the corresponding integrable cases in the Euler equations on $s o^{*}(4)$. Now we recover this bivector by solving equations (3.1), (3.2) and (3.3) for the Steklov-Lyapunov system.

Let us briefly discuss the bi-Hamiltonian structure related with the Kötter variables of separation. Elliptic coordinates on the sphere $\mathbb{S}^{2}(2.13)$ are associated with the polynomial Poisson bivector

$$
P_{\text {ell }}^{\prime}=\mathcal{L}_{X} P,
$$

where $\mathcal{L}_{X}$ is a Lie derivative along the vector field $X=\sum X^{j} \partial_{j}$ with the following entries:

$$
X^{i}=0, \quad X^{i+3}=[x \times \mathbf{A}(x \times J)]_{i}, \quad i=1,2,3 .
$$

Bivector $P_{e}^{\prime}$ is compatible with $P$ and has the same foliation by symplectic leaves as $P$.

Using the Poisson map (2.9), (2.10) we can easily express $P_{\text {ell }}^{\prime}$ in the initial variables $p, M$. It will be a rational bivector $P_{\text {ell }}^{\prime}=R / Q$, where $R$ is a bivector with fourth-order homogeneous polynomial entries and $Q=|(M-\mathbf{B} p) \times p|^{2}$ is a fourth-order polynomial as well. So, we could directly calculate the Kötter variables solving equations (3.1), (3.2) and (3.3) in the corresponding space of rational bivectors.

\subsection{Calculation of variables of separation}

The bi-involutivity of the integrals of motion (3.1) is equivalent to the existence of control matrix $F$ defined by

$$
P^{\prime} d H=P(F d H), \quad \text { or } \quad P^{\prime} d H_{i}=P \sum_{j=1}^{n} F_{i j} d H_{j}, \quad i=1, \ldots, n .
$$

The additional assumption (3.3) ensures that $F$ is a non-degenerate matrix and the eigenvalues of $F$ are the desired variables of separation [20,21]. Moreover, for the so-called Stäckel separable systems the suitable normalized left eigenvectors of the control matrix $F$ form the Stäckel matrix $S[20,21,22,24]$. In this case separated relations (1.1) are affine equations in integrals of motion $H_{k}$.

Let us calculate the control matrices for the Steklov-Lyapunov system and, for brevity, introduce three constants

$$
\tau_{k}=\operatorname{tr} \mathbf{A}^{k} \equiv \sum_{i=1}^{3} a_{i}^{k}, \quad k=0,1,2,
$$


and some linear functions on variables $p, M$

$$
\rho_{k}=\left\langle c, \mathbf{A}^{k} p\right\rangle, \quad \sigma_{k}=\left\langle c, \mathbf{A}^{k} M\right\rangle, \quad k=0,1,2,
$$

which are related to each other via the Casimir functions (2.3) on $e^{*}(3)$. For instance,

$$
C_{1}=\sum_{i=1}^{3} p_{i}^{2}=-\frac{1}{2}\left(\tau_{1}^{2}-\tau_{2}\right) \rho_{0}^{2}+2\left(\tau_{1} \rho_{1}-\rho_{2}\right) \rho_{0}-\rho_{1}^{2} .
$$

In this notations control matrices associated with the Hamiltonians $H_{1,2}(2.7)$ and the Poisson bivectors $P_{1,2}^{\prime}(3.4)$, (3.5) look like

$$
F_{1}=\left(\begin{array}{cc}
-2 \rho_{1} & 2 \rho_{0} \\
\sigma_{1}+\rho_{2}-\tau_{1} \rho_{1} & -\sigma_{0}-\rho_{1}+\tau_{1} \rho_{0}
\end{array}\right), \quad F_{2}=\left(\begin{array}{cc}
\frac{\sigma_{0}-\rho_{1}}{\rho_{0}}-\frac{\tau_{1}}{3} & 2 \\
\frac{\sigma_{1}+\rho_{2}-\tau_{1} \rho_{1}}{\rho_{0}} & \frac{2 \tau_{1}}{3}
\end{array}\right) .
$$

Now we can simply calculate the desired variables of separation using two control matrices. Namely, let $u_{1,2}$ be eigenvalues of the control matrix $F_{2}$

$$
\begin{aligned}
B(\lambda) & =\operatorname{det}\left(F_{2}-\lambda\right)=\left(\lambda-u_{1}\right)\left(\lambda-u_{2}\right) \\
& =\lambda^{2}-\left(\frac{\sigma_{0}-\rho_{1}}{\rho_{0}}+\frac{\tau_{1}}{3}\right) \lambda-\frac{2 \tau_{1}^{2}}{9}+\frac{2\left(2 \rho_{1}+\sigma_{0}\right) \tau_{1}}{3 \rho_{0}}-\frac{2\left(\sigma_{1}+\rho_{2}\right)}{\rho_{0}},
\end{aligned}
$$

whereas the eigenvalues of $F_{1}$ be doubled momenta $2 p_{u_{1,2}}$, so that the characteristic polynomial has the form

$$
\begin{aligned}
A(\lambda) & =\operatorname{det}\left(F_{1}-\lambda\right)=\left(\lambda-2 p_{u_{1}}\right)\left(\lambda-2 p_{u_{2}}\right) \\
& =\lambda^{2}+\left(\sigma_{0}+3 \rho_{1}-\tau_{1} \rho_{0}\right) \lambda+2 \rho_{1}\left(\sigma_{0}+\rho_{1}\right)-2 \rho_{0}\left(\sigma_{1}+\rho_{2}\right) .
\end{aligned}
$$

Another equivalent definition of momenta $p_{u_{i}}$ is given by a relation

$$
p_{u_{i}}=\frac{\langle c, p\rangle}{2} u_{i}-\frac{\langle c, M\rangle}{2}+\frac{\langle c, d \times p\rangle}{3}, \quad i=1,2 .
$$

Now we can prove the following

Proposition 5. On symplectic leaves of $e^{*}(3)$ variables $u_{1,2}$ and $p_{u_{1,2}}$ are canonical variables

$$
\left\{u_{i}, p_{u_{i}}\right\}=1, \quad\left\{u_{i}, p_{u_{i}}\right\}_{1}^{\prime}=2 p_{u_{i}}, \quad\left\{u_{i}, p_{u_{i}}\right\}_{2}^{\prime}=u_{i}, \quad i=1,2 .
$$

with respect to canonical Poisson bracket (2.2).

The proof consists of the calculation of the Poisson brackets between coefficients of characteristic polynomials $A(\lambda)(3.13)$ and $B(\lambda)(3.12)$.

Now we have to determine an inverse transformation from variables $u_{1,2}$ and $p_{u_{1,2}}$ to initial variables $p, M$. Firstly, using the definitions (3.13), (3.12) and the relation (3.10), we express five linear functions (3.9) via variables of separation and Casimir functions

$$
\begin{aligned}
& \rho_{0}=2 \frac{p_{u_{1}}-p_{u_{2}}}{u_{1}-u_{2}}, \quad \rho_{1}=\frac{2 \tau_{1}-3 u_{1}}{3\left(u_{1}-u_{2}\right)} p_{u_{1}}-\frac{2 \tau_{1}-3 u_{2}}{3\left(u_{1}-u_{2}\right)} p_{u_{2}}, \\
& \rho_{2}=\frac{\left(u_{1}-u_{2}\right) C_{1}}{4\left(p_{u_{1}}-p_{u_{2}}\right)}-\left(\tau_{2}+\frac{\tau_{1}^{2}}{9}\right) \frac{p_{u_{1}}-p_{u_{2}}}{2\left(u_{1}-u_{2}\right)}+\frac{2 \tau_{1}}{3} \frac{p_{u_{1}} u_{2}-p_{u_{2}} u_{1}}{u_{1}-u_{2}}+\frac{\left(p_{u_{1}} u_{2}-p_{u_{2}} u_{1}\right)^{2}}{4\left(p_{u_{1}}-p_{u_{2}}\right)\left(u_{1}-u_{2}\right)}, \\
& \sigma_{0}=\frac{u_{1}+2 u_{2}}{u_{1}-u_{2}} p_{u_{1}}-\frac{2 u_{1}+u_{2}}{u_{1}-u_{2}} p_{u_{2}}, \\
& \sigma_{1}=-\rho_{2}+\frac{\tau_{1} \rho_{1}}{3}-\frac{2\left(p_{u_{1}} u_{1}-p_{u_{2}} u_{2}\right) \tau_{1}}{3\left(u_{1}-u_{2}\right)}+\frac{\left(p_{u_{1}}-p_{u_{2}}\right) u_{1} u_{2}}{u_{1}-u_{2}} .
\end{aligned}
$$

It is easy to see that $\rho_{k}$ and $\sigma_{k}$ are symmetric functions in $u_{1,2}, p_{u_{1,2}}$. 
Secondly, we determine the initial variables $p, M$ as functions on $\rho_{k}$ and $\sigma_{k}$ :

$$
\begin{aligned}
& p_{i}=c_{i}\left(a_{j} a_{k} \rho_{0}-\left(a_{j}+a_{k}\right) \rho_{1}+\rho_{2}\right), \quad i=1,2,3, \quad(i, j, k)=(1,2,3), \\
& M_{i}=\frac{c_{i}}{\rho_{0}}\left(C_{2}-\sigma_{1}\left(a_{i} \rho_{0}-\rho_{1}\right)+\sigma_{0}\left(\rho_{2}-\tau_{1} \rho_{1}+a_{i}\left(a_{j}+a_{k}\right) \rho_{0}\right)\right) .
\end{aligned}
$$

Here $c_{i}$ are entries of the vector $c(3.8), C_{1,2}$ are the Casimir functions on $e^{*}(3)(2.3)$ and $(i, j, k)$ means the permutation of $(1,2,3)$.

Matrices $F_{1,2}(3.11)$ in canonical variables of separation look like

$$
F_{1}=S\left(\begin{array}{cc}
2 p_{1} & 0 \\
0 & 2 p_{2}
\end{array}\right) S^{-1}, \quad F_{2}=S\left(\begin{array}{cc}
q_{1} & 0 \\
0 & q_{2}
\end{array}\right) S^{-1}
$$

where the Stäckel matrix $S$ is equal to

$$
S=\left(\begin{array}{cc}
\frac{6}{2 \tau_{1}-3 u_{1}} & \frac{6}{2 \tau_{1}-3 u_{2}} \\
1 & 1
\end{array}\right)
$$

The notion of the Stäckel matrix $S$ allows us to easily get the separated relations (1.1) and prove that canonical variables $u, p_{u}$ are the variables of separation for the Steklov-Lyapunov system.

Proposition 6. In the Steklov-Lyapunov case the canonical variables $u_{1,2}(3.12)$ and $p_{u_{1,2}}(3.13)$ satisfy the following separated relations

$$
\Phi\left(u, p_{u}\right)=\left(\frac{u}{2}-\frac{\tau_{1}}{3}\right) H_{1}+H_{2}+\varphi_{3}(u) p_{u}^{2}+\phi_{3}(u)=0, \quad u=u_{1,2}, \quad p_{u}=p_{u_{1,2}},
$$

where cubic polynomials $\varphi_{3}(u)$ and $\phi_{3}(u)$ are equal to

$$
\begin{aligned}
\varphi_{3}(u)= & \left(\frac{u^{3}}{2}+\frac{u}{3}\left(\tau_{1}^{2}-3 \tau_{2}\right)-\frac{4}{27}\left(\tau_{1}-3 a_{1}\right)\left(\tau_{1}-3 a_{2}\right)\left(\tau_{1}-3 a_{3}\right)\right), \\
\phi_{3}(u)= & \frac{C_{1} u^{3}}{2}+C_{2} u^{2}-\left(\frac{C_{2} \tau_{1}+C_{1} \tau_{1}^{2}}{3}-\frac{C_{1} \tau_{2}}{2}\right) u+C_{2}\left(\frac{7 \tau_{1}^{2}}{9}-\tau_{2}\right) \\
& +C_{1}\left(\frac{\tau_{1}^{3}}{27}+\frac{2 \tau_{1} \tau_{2}}{3}-\frac{2 \tau_{3}}{3}\right) .
\end{aligned}
$$

The proof consists of substituting integrals of motion $H_{1,2}(2.7)$ in terms of variables of separation (3.15) into the separated relations (3.17).

So, in the Steklov-Lyapunov case equations of motion are linearized on Jacobian of the genus two hyperelliptic curve defined by the equation $\Phi\left(u, p_{u}\right)=0(3.17)$ and the system of the Abel-Jacobi equations has the standard form

$$
\begin{aligned}
& \int_{\infty}^{u_{1}} \frac{d u}{p(u) \varphi_{3}(u)}+\int_{\infty}^{u_{2}} \frac{d u}{p(u) \varphi_{3}(u)}=\beta_{1} t+\gamma_{1}, \\
& \int_{\infty}^{u_{1}} \frac{u d u}{p(u) \varphi_{3}(u)}+\int_{\infty}^{u_{2}} \frac{u d u}{p(u) \varphi_{3}(u)}=\beta_{2} t+\gamma_{2} .
\end{aligned}
$$

Here $p(u)$ means the function $p_{u}$ on $u$ obtained from the equation (3.17), $\beta_{1,2}$ are certain constants depending only on the choice of the Hamiltonian $\left(H_{1}\right.$ or $\left.H_{2}\right)$ and $\gamma_{1,2}$ are two constants. Solving these equations with respect to $u_{1,2}\left(t, \gamma_{1,2}\right)$ and substituting these solutions into the expressions (3.14) and (3.15) we finally get the initial variables $p, M$ as functions on time variable $t$ and six constants $H_{1,2}, C_{1,2}$ and $\gamma_{1,2}$. 
Remark 5. In order to give an explicit theta-functions solution, one can apply the standard machinery of the Weierstrass root functions describing inversion of the hyperelliptic quadratures (3.19), completely similar to solution of Jacobi's geodesic problem or Neumann's particular case of the Clebsch system $[5,8,27]$.

Using shift of coordinates

$$
u_{i} \rightarrow \frac{2}{3}\left(a_{1}+a_{2}+a_{3}-3 u_{i}\right)
$$

we can rewrite separated relations (3.17) in the following form

$$
p_{u}^{2}=\frac{C_{1} u^{3}+\left(C_{1} \operatorname{tr} \mathbf{A}-C_{2}\right) u^{2}+4 \tilde{H}_{1} u+4 \tilde{H}_{2}}{\operatorname{det}(u-\mathbf{A})},
$$

where

$$
\tilde{H}_{1}=-H_{1}-C_{1} \operatorname{tr}\left(\mathbf{A}^{2}+4 \mathbf{A}^{\vee}\right)+2 C_{2} \operatorname{tr} \mathbf{A}, \quad \tilde{H}_{2}=H_{2}+2 C_{1} \operatorname{det} \mathbf{A}-2 C_{2} \operatorname{tr} \mathbf{A}^{\vee} .
$$

Separated relations (3.20) have the same form as the Kötter separated relations (2.14). However, we have to point out that if $p$ and $M$ are real variable, then $u_{1,2}$ are complex functions in contrast with the real Kötter variables $v_{1,2}$. In terms of $u_{1,2}$ and $p_{u_{1,2}}$ symmetric functions on the Kötter variables $v_{1,2}$ look like

$$
v_{1}+v_{2}=\frac{P\left(u_{1}, u_{2}, p_{u_{1}}, p_{u_{2}}\right)}{Q\left(u_{1}, u_{2}, p_{u_{1}}, p_{u_{2}}\right)}, \quad v_{1} v_{2}=\frac{R\left(u_{1}, u_{2}, p_{u_{1}}, p_{u_{2}}\right)}{T\left(u_{1}, u_{2}, p_{u_{1}}, p_{u_{2}}\right)} .
$$

Here $P, Q, R, T$ are the sixth-order polynomials in momenta, $Q, T$ are the sixth-order polynomials in coordinates, whereas $P$ and $R$ are the seventh- and eighth-order polynomials in coordinates. We did not find a foreseeable expressions for these polynomials or their combinations. In any case variables $u, p_{u}$ and $v, p_{v}$ are related by non-trivial canonical transformation (1.3).

Remark 6. The Steklov-Lyapunov system on $e^{*}(3)$ coincides with the Steklov system on $s o^{*}(4)$ after some linear change of phase variables [3]. It is a twisted Poisson map, which permutes first and second Lie-Poisson brackets on $e^{*}(3)$ and $s o^{*}(4)$ [19].

We suppose that variables $u_{1,2}$ and $p_{u_{1,2}}$ coincide with the complex variables of separation for the Steklov system on $s o(4)$ introduced in $[6,13]$ up to this change of variables and transformation of the canonical momenta associated with permutation of the Poisson brackets. We thank one of the referees for the reference on these papers. Algebro-geometric relations of this complex coordinates $u_{1,2}$ with the real Kötter coordinates $v_{1,2}$ is discussed in [8].

\subsection{The Rubanovsky system}

Let us consider a nontrivial integrable generalization of the Steklov-Lyapunov system discovered by Rubanovsky [16]

$$
\hat{H}_{1}=H_{1}+2\langle b, p\rangle, \quad \hat{H}_{2}=H_{2}+\langle b,(\operatorname{tr} \mathbf{A}-\mathbf{A}) p\rangle-\langle b, M\rangle,
$$

where $H_{1,2}$ are given by $(2.7)$ and $b=\left(b_{1}, b_{2}, b_{3}\right)$ is a constant vector. This deformation describes the motion of a gyrostat in an ideal fluid under the action of the Archimedes torque, which arises when the barycenter of the gyrostat does not coincide with its volume center. The problem of separation of variables for the Rubanovsky systems was unsolved up until now.

The Rubanovsky integrals of motion are non-homogeneous second-order polynomials and, therefore, it is natural to solve the equations (3.1) and (3.2) in the space of non-homogeneous second-order polynomial bivectors and in the similar space of rational bivectors. 
Proposition 7. Integrals of motion $\hat{H}_{1,2}(p, M)$ (3.21) are in bi-involution (3.1) with respect to the Poisson brackets associated with the polynomial Poisson bivector

$$
\hat{P}_{1}^{\prime}=P_{1}^{\prime}+\langle b, c\rangle\left(\begin{array}{cccccc}
0 & 0 & 0 & 0 & 0 & 0 \\
0 & 0 & 0 & 0 & 0 & 0 \\
0 & 0 & 0 & 0 & 0 & 0 \\
0 & 0 & 0 & 0 & p_{3} & -p_{2} \\
0 & 0 & 0 & -p_{3} & 0 & p_{1} \\
0 & 0 & 0 & p_{2} & -p_{1} & 0
\end{array}\right)
$$

and the rational Poisson bivector

$$
\hat{P}_{2}^{\prime}=\frac{1}{\langle c, p\rangle} \hat{P}_{1}^{\prime}+\frac{1}{\langle c, p\rangle}\left(\langle c, M\rangle+\frac{\alpha\left(1+\alpha^{2}\langle c, d\rangle\right)}{2 \alpha^{2}\langle c, d\rangle}\langle c \times p, d\rangle\right) P,
$$

where $P_{1}^{\prime}$ is the Poisson bivector for the Steklov-Lyapunov system (3.4) in which $\alpha=1$ and vectors $c$ and $d$ are given by (3.8).

The proof is a straightforward verification of the equations (3.1) and (3.2).

Remark 7. If $c=\left(c_{1}, c_{2}, \sqrt{-c_{1}^{2}-c_{2}^{2}}\right)$ is an arbitrary vector, $d=\left(c_{1} \alpha^{-2}, 0,0\right)$ and $\alpha \rightarrow \infty$, then the Poisson bivectors $\hat{P}_{1,2}^{\prime}$ yield bi-Hamiltonian structures on $e^{*}(3)$ associated with the Lagrange top [20].

Following the same line as previously, let us calculate the control matrices associated with the Hamiltonians $\hat{H}_{1,2}(3.21)$ and the Poisson bivectors $\hat{P}_{1,2}^{\prime}(3.22),(3.23)$

$$
\hat{F}_{1}=F_{1}-\frac{\langle b, c\rangle}{2}\left(\begin{array}{cc}
0 & 0 \\
1 & 0
\end{array}\right), \quad \hat{F}_{2}=F_{2}-\frac{\langle b, c\rangle}{2 \rho_{0}}\left(\begin{array}{ll}
0 & 0 \\
1 & 0
\end{array}\right) .
$$

Eigenvalues of these matrices are new canonical variables of separation $\hat{u}_{1,2}$ and $\hat{p}_{u_{1,2}}$, so that

$$
\hat{F}_{1}=S\left(\begin{array}{cc}
2 \hat{p}_{1} & 0 \\
0 & 2 \hat{p}_{2}
\end{array}\right) S^{-1}, \quad \hat{F}_{2}=S\left(\begin{array}{cc}
\hat{u}_{1} & 0 \\
0 & \hat{u}_{2}
\end{array}\right) S^{-1}
$$

where $S$ is the same Stäckel matrix (3.16). These variables are simply related with the previous one

$$
\begin{aligned}
& \hat{u}_{1,2}=\frac{u_{1}+u_{2}}{2} \pm \frac{1}{2} \sqrt{\left(u_{1}-u_{2}\right)^{2}-\frac{4\langle b, c\rangle}{\rho_{0}}}, \\
& \hat{p}_{u_{1,2}}=\frac{p_{u_{1}}+p_{u_{2}}}{2} \pm \frac{1}{2} \sqrt{\left(p_{u_{1}}-p_{u_{2}}\right)^{2}-\langle b, c\rangle \rho_{0}} .
\end{aligned}
$$

Initial variables $p, M$ are the same functions on $\hat{\rho}_{k}$ and $\hat{\sigma}_{k}$

$$
\begin{aligned}
& p_{i}=c_{i}\left(a_{j} a_{k} \hat{\rho}_{0}-\left(a_{j}+a_{k}\right) \hat{\rho}_{1}+\hat{\rho}_{2}\right), \quad i=1,2,3, \quad(i, j, k)=(1,2,3), \\
& M_{i}=\frac{c_{i}}{\hat{\rho}_{0}}\left(C_{2}-\hat{\sigma}_{1}\left(a_{i} \hat{\rho}_{0}-\hat{\rho}_{1}\right)+\hat{\sigma}_{0}\left(\hat{\rho}_{2}-\tau_{1} \hat{\rho}_{1}+a_{i}\left(a_{j}+a_{k}\right) \hat{\rho}_{0}\right)\right),
\end{aligned}
$$

where four functions $\hat{\rho}_{k}\left(\hat{u}, \hat{p}_{u}\right)=\rho_{k}(u, p)$ and $\hat{\sigma}_{0}\left(\hat{u}, \hat{p}_{u}\right)=\sigma_{0}\left(\hat{u}, \hat{p}_{u}\right)$ is given by $(3.14)$ :

$$
\begin{aligned}
& \hat{\rho}_{0}=2 \frac{\hat{p}_{u_{1}}-\hat{p}_{u_{2}}}{\hat{u}_{1}-\hat{u}_{2}}, \quad \hat{\rho}_{1}=\frac{2 \tau_{1}-3 \hat{u}_{1}}{3\left(\hat{u}_{1}-\hat{u}_{2}\right)} \hat{p}_{u_{1}}-\frac{2 \tau_{1}-3 \hat{u}_{2}}{3\left(\hat{u}_{1}-\hat{u}_{2}\right)} \hat{p}_{u_{2}}, \\
& \hat{\sigma}_{0}=\frac{\hat{u}_{1}+2 \hat{u}_{2}}{\hat{u}_{1}-\hat{u}_{2}} \hat{p}_{u_{1}}-\frac{2 \hat{u}_{1}+\hat{u}_{2}}{\hat{u}_{1}-\hat{u}_{2}} \hat{p}_{u_{2}}, \\
& \hat{\rho}_{2}=\frac{\left(\hat{u}_{1}-\hat{u}_{2}\right) C_{1}}{4\left(\hat{p}_{u_{1}}-\hat{p}_{u_{2}}\right)}-\left(\tau_{2}+\frac{\tau_{1}^{2}}{9}\right) \frac{\hat{p}_{u_{1}}-\hat{p}_{u_{2}}}{2\left(\hat{u}_{1}-\hat{u}_{2}\right)}+\frac{2 \tau_{1}}{3} \frac{\hat{p}_{u_{1}} \hat{u}_{2}-\hat{p}_{u_{2}} \hat{u}_{1}}{\hat{u}_{1}-\hat{u}_{2}}+\frac{\left(\hat{p}_{u_{1}} \hat{u}_{2}-\hat{p}_{u_{2}} \hat{u}_{1}\right)^{2}}{4\left(\hat{p}_{u_{1}}-\hat{p}_{u_{2}}\right)\left(\hat{u}_{1}-\hat{u}_{2}\right)}
\end{aligned}
$$


and one function is different

$$
\hat{\sigma}_{1}=\frac{\langle b, c\rangle}{2}-\hat{\rho}_{2}+\frac{\tau_{1} \hat{\rho}_{1}}{3}-\frac{2\left(\hat{p}_{u_{1}} \hat{u}_{1}-\hat{p}_{u_{2}} \hat{u}_{2}\right) \tau_{1}}{3\left(\hat{u}_{1}-\hat{u}_{2}\right)}+\frac{\left(\hat{p}_{u_{1}}-\hat{p}_{u_{2}}\right) \hat{u}_{1} \hat{u}_{2}}{\hat{u}_{1}-\hat{u}_{2}} .
$$

This shift of $\hat{\sigma}_{1}$ acts only on $M$ variables (3.15).

Proposition 8. In the Rubanovski case the separated relations have the following form

$$
\begin{aligned}
\hat{\Phi}\left(\hat{u}, \hat{p}_{u}\right)= & \left(\frac{\hat{u}}{2}-\frac{\tau_{1}}{3}\right) \hat{H}_{1}+\hat{H}_{2}+\varphi_{3}(\hat{u}) \hat{p}_{u}^{2}-\left(\frac{\langle b, c\rangle}{2} \hat{u}^{2}+\frac{\langle b, \mathbf{D} c\rangle}{3} \hat{u}+\frac{2\left\langle b, \mathbf{D}^{\vee} c\right\rangle}{9}\right) \hat{p}_{u} \\
& +\phi_{3}(\hat{u})-\frac{\langle b, c\rangle}{2}\left(\frac{\hat{u}}{4}+\frac{\tau_{1}}{2}\right)+\frac{\langle b, c\rangle\langle b, \mathbf{A} c\rangle}{2}=0, \quad \hat{u}=\hat{u}_{1,2}, \quad \hat{p}_{u}=\hat{p}_{u_{1,2}} .
\end{aligned}
$$

Here cubic polynomials $\varphi_{3}$ and $\phi_{3}$ are given by (3.18) and $\mathbf{D}=\operatorname{tr} \mathbf{A}-3 \mathbf{A}$.

The proof consists of substituting integrals of motion $\hat{H}_{1,2}$ in terms of variables of separation $\hat{u}, \hat{p}_{u}$ into the separated relations (3.24).

As above, we can prove that the equations of motion are linearized on the Jacobian variety of the genus two hyperelliptic curve defined by (3.24). For the brevity, here we omit the explicit expressions for a base of holomorphic differentials and the corresponding Abel-Jacobi equations, which may be easily obtained using modern computer algebra software.

Calculation of the separating variables and the corresponding algebraic curve for the $\mathrm{Ru}-$ banovski gyrostat is a new result, which allows us to make conclusion an applicability of biHamiltonian methods to study finite-dimensional integrable Hamiltonian systems.

\section{Acknowledgements}

The author is grateful to the referees for a number of helpful suggestions that resulted in improvement of the article.

\section{References}

[1] Adler M., van Moerbeke P., Vanhaecke P., Algebraic integrability, Painlevé geometry and Lie algebras, A Series of Modern Surveys in Mathematics, Vol. 47, Springer-Verlag, Berlin, 2004.

[2] Belokolos E.D., Bobenko A.I., Enol'skii V.Z., Its A.R., Matveev V.B., Algebro-geometric approach to nonlinear integrable equations, Springer Series in Nonlinear Dynamics, Springer-Verlag, Berlin, 1994.

[3] Bobenko A.I., Euler equations on the algebras e(3) and so(4). Isomorphism of the integrable cases, Funct. Anal. Appl. 20 (1986), 53-56.

[4] Bolsinov A.V., Fedorov Y.N., Steklov-Lyapunov type systems, Preprint, 2003, available at http:// upcommons . upc.edu/e-prints/bitstream/2117/900/4/0303f edorov.pdf.

[5] Borisov A.V., Tsiganov A.V. (Editors), Klebsch system. Separation of variables, explicit integration?, RCD, Moscow - Izhevsk, 2009.

[6] Bueken P., Vanhaecke P., The moduli problem for integrable systems: the example of a geodesic flow on SO(4), J. London Math. Soc. 62 (2000), 357-369.

[7] Falqui G., Pedroni M., Separation of variables for bi-Hamiltonian systems, Math. Phys. Anal. Geom. 6 (2003), 139-179, nlin.SI/0204029.

[8] Fedorov Y., Basak I., Separation of variables and explicit theta-function solution of the classical SteklovLyapunov systems: a geometric and algebraic geometric background, Regul. Chaotic Dyn. 16 (2011), 374395, arXiv:0912.1788.

[9] Kirchhoff G.R., Vorlesungen über mathematische Physik Mechanik, Leipzig, 1874.

[10] Kolosoff G.V., Sur le mouvement d'un corp solide dans un liquide indéfini, C.R. Acad. Sci. Paris 169 (1919), 685-686. 
[11] Kötter F., Die von Steklow und Liapunow entdeckten integralen Fälle der Bewegung eines starren Körpers in einer Flüssigkeit, Sitzungsber. König. Preuss. Akad. Wiss. 6 (1900), 79-87.

[12] Kötter F., Über die Bewegung eines festen Körpers in einer Flussigkeit, J. für Math. 109 (1892), 51-81, 89-111.

[13] Kuznetsov V., Vanhaecke P., Bäcklund transformations for finite-dimensional integrable systems: a geometric approach, J. Geom. Phys. 44 (2002), 1-40, nlin.SI/0004003.

[14] Lyapunov A.M., New integrable case of the equations of motion of a rigid body in a fluid, Fortschr. Math. 25 (1897), 1501-1504.

[15] Novikov S.P., Shmel'tser I., Periodic solutions of Kirchhoff equations for the free motion of a rigid body in a fluid and the extended Lyusternik-Shnirel'man-Morse theory (LSM). I, Funct. Anal. Appl. 15 (1981), 197-207.

[16] Rubanovsky V.N., Integrable cases in the problem of a heavy solid moving in a fluid, Dokl. Akad. Nauk SSSR 180 (1968), 556-559.

[17] Stekloff W., Ueber die Bewegung eines festen Körpers in einer Flüssigkeit, Math. Ann. 42 (1893), 273-274.

[18] Tsiganov A.V., New variables of separation for particular case of the Kowalevski top, Regul. Chaotic Dyn. 15 (2010), 659-669, arXiv:1001.4599.

[19] Tsiganov A.V., On an isomorphism of integrable cases of the Euler equations on the bi-Hamiltonian manifolds e(3) and so(4), J. Math. Sci. 136 (2006), 3641-3647.

[20] Tsiganov A.V., On bi-Hamiltonian geometry of the Lagrange top, J. Phys. A: Math. Theor. 41 (2008), 315212, 12 pages, arXiv:0802.3951.

[21] Tsiganov A.V., On bi-Hamiltonian structure of some integrable systems on so*(4), J. Nonlinear Math. Phys. 15 (2008), 171-185, nlin.SI/0703062.

[22] Tsiganov A.V., On bi-integrable natural Hamiltonian systems on Riemannian manifolds, J. Nonlinear Math. Phys. 18 (2011), 245-268, arXiv:1006.3914.

[23] Tsiganov A.V., On isomorphism of the Steklov-Lyapunov system with the potential motion on the sphere, Dokl. Math. 71 (2005), 145-147.

[24] Tsiganov A.V., On natural Poisson bivectors on the sphere, J. Phys. A: Math. Theor. 44 (2011), 105203, 21 pages, arXiv:1010.3492.

[25] Tsiganov A.V., On the Steklov-Lyapunov case of the rigid body motion, Regul. Chaotic Dyn. 9 (2004), 77-89, nlin.SI/0406017.

[26] Tsiganov A.V., On two different bi-Hamiltonian structures for the Toda lattice, J. Phys. A: Math. Gen. 40 (2007), 6395-6406, nlin.SI/0701062.

[27] Weierstrass K., Mathematische Werke I, Mayer \& Muller, Berlin, 1894. 